\title{
Knockdown of KLK11 inhibits cell proliferation and increases oxaliplatin sensitivity in human colorectal cancer
}

\author{
ZONGBIN XU ${ }^{1}$, PAN CHI $^{1}$, JIE PAN $^{2}$, SONGFEI SHEN ${ }^{3}$, YANWU SUN ${ }^{1}$, \\ XIAOJIE WANG ${ }^{1}$ and XINGRONG LU ${ }^{1}$ \\ Departments of ${ }^{1}$ Colorectal Surgery, ${ }^{2}$ Emergency Surgery and ${ }^{3}$ Medical Oncology, Union Hospital, \\ Fujian Medical University, Fuzhou, Fujian 350001, P.R. China
}

Received June 23, 2015; Accepted August 11, 2016

DOI: $10.3892 /$ etm.2016.3723

\begin{abstract}
It has been reported that kallikrein 11 (KLK11) is crucially involved in the development and progression of various types of cancer. However, the molecular mechanisms that underlie the involvement of KLK11 in aberrant colorectal cancer (CRC) cell growth remain largely unclear. The aim of the present study was to investigate the role of KLK11 and the effects of KLK11 on oxaliplatin (L-OHP) chemosensitivity by knocking down KLK11 in LOVO and HCT-8 cells. Loss-of-function assays revealed KLK11 inhibition significantly inhibited growth and induced apoptosis of CRC cells in vitro. Notably, further experiments found that knockdown of KLK11 expression increased the L-OHP chemosensitivity of CRC cells. KLK11 inhibition of increased L-OHP-induced apoptosis may be associated with activation of caspase-3 cleavage and the apoptosis signaling pathway. The present results indicated that KLK11 may be an potential target of interest for future research into therapies for CRC.
\end{abstract}

\section{Introduction}

Colorectal cancer (CRC) is among the most common malignancies and the second leading cause of cancer-associated mortality, following lung cancer (1). The 5-year survival rate for CRC is still low because patients diagnosed with CRC have progressed to the advanced stage (2-5). Survival rates have increased with the introduction of irinotecan and oxaliplatin chemotherapy, as well as the use of targeted therapies in the past decade (6-8). Combined perioperative chemotherapy and surgery is a major therapeutic treatment for patients

Correspondence to: Professor Xingrong Lu, Department of Colorectal Surgery, Union Hospital, Fujian Medical University, 29 Xinquan Road, Fuzhou, Fujian 350001, P.R. China

E-mail: luxingrong2015@163.com

Key words: colorectal cancer, kallikrein 11, oxaliplatin, drug resistance, apoptosis with initially resectable liver metastases from CRC (9-11). However, the development of drug resistance in cancer cells raises a major challenge to chemotherapy and restricts the anticancer efficacy of chemotherapeutic drugs (12-14). Therefore, improving the sensitivity to drug resistance remains an urgent requirement for chemoresistance.

Human kallikrein 11 (KLK11) is a member of the human $K L K$ gene family and located at the chromosomal locus 19q13.3-q13.4 (15). Previous experiments have indicated that KLK11 is ubiquitously expressed in human brain, skin, stomach, breast, prostate, ovary and intestine tissue (16). Recent results demonstrated that KLKs were involved in human malignancies and that KLK11 may be a favorable prognostic biomarker for ovarian and prostate cancer due to the high serum levels of KLK11 in $70 \%$ of women with ovarian cancer and in $60 \%$ of men with prostate cancer (17). Alexopoulou et al have shown that KLK11 mRNA expression was upregulated in colorectal adenocarcinoma and could be considered as a new molecular prognostic biomarker (18). However, the value of KLK11 as a prognostic biomarker remains controversial and more evidence is needed for further clinical application. It has been reported that KLK11 mRNA expression could serve as a novel and independent biomarker for diagnosis and prognosis in laryngeal cancer (19). Unal et al have suggested that KLK11-positive patients had higher disease-free survival and overall survival compared to those with KLK11-negative expression (20). However, little is known concerning the possible involvement of KLK11 in human CRC.

The aim of the present study was to investigate the role of KLK11 in human CRC. Additionally, the potential use of shRNA-mediated KLK11 gene knockdown associated with apoptosis and drug resistance were further examined.

\section{Materials and methods}

Cell culture and reagents. Two human-derived CRC cell lines LOVO (CCL-229) and HCT-8 (CCL-244) were obtained from the American Type Culture Collection (Manassas, VA, USA) and cultured with RPMI-1640 (Invitrogen; Thermo Fisher Scientific, Inc., Carlsbad, CA, USA) supplemented with $10 \%$ fetal bovine serum (FBS; Invitrogen), $100 \mathrm{U} / \mathrm{ml}$ penicillin 
and $100 \mathrm{mg} / \mathrm{ml}$ streptomycin (Thermo Fisher Scientific, Inc., Waltham, MA, USA) in $5 \% \mathrm{CO}_{2}$ at $37^{\circ} \mathrm{C}$.

Reverse transcription-quantitative polymerase chain reaction (RT-qPCR) assay. Total RNA from cells was isolated using TRIzol reagent (Invitrogen) according to the manufacturer's directions. Then, $1 \mu \mathrm{g}$ total RNA was used for reverse transcription reaction using SuperScript III reverse transcriptase (Invitrogen). qPCR was performed using an ABI 7500 real-time PCR system (Applied Biosystems; Thermo Fisher Scientific, Inc., Foster City, CA, USA), and the mRNA expression of human KLK11 and $\beta$-actin was evaluated using a LightCycler Fast Start DNA Master SYBR Green I kit (Roche Diagnostics $\mathrm{GmbH}$, Mannheim, Germany). PCR amplification was performed by denaturation at $95^{\circ} \mathrm{C}$ for $10 \mathrm{~min}$, annealing and extension at $60^{\circ} \mathrm{C}$ for $60 \mathrm{sec}$ for 40 cycles. RT-qPCR analysis was performed using the following primers: KLK11 forward: 5'-GTTCGAGAAGACGCGGCTAC-3'; KLK11 reverse: 5'-GGTGGGAGAGGTGAGTGAC-3'. $\beta$-actin forward: 5-CCAACCGCGAGAAGATGA-3'; $\beta$-actin reverse: 5'-CCA GAGGCGTACAGGGATAG-3'. The relative expression level of KLK11 was calculated using the $\Delta \Delta C q$ method (21) and normalized against that of $\beta$-actin. All PCR amplification was performed in triplicate and repeated in three independent experiments.

Gene silencing with the lentivirus encoding specific shRNA. In order to silencing KLK11, the short hairpin RNA (shRNA) were generated by ligating synthetic oligonucleotides (Invitrogen) against the target genes into the AgeI and EcoRI sites of pLKO.1-TRC cloning vector (provided by Dr Xuchao Zhu; Tenth People's Hospital, Affiliated to Tongji University, Shanghai, China). The sequences of the KLK11 shRNA (shKLK11) and shRNA control (SCR) were as follows: KLK11-SH1 sense, 5'-CCGGCCAACAAAGACCACCGC AATGCTCGAGCATTGCGGTGGTCTTTGTTGGTTTTT G-3' and antisense, 5'-AATTCAAAAACCAACAAAGAC CACCGCAATGCTCGAGCATTGCGGTGGTCTTTG TTGG-3'; KLK11-SH2 sense, 5'-CCGGGAGATGCTGTC ACTTAATAATCTCGAGATTATTAAGTGACAGCATCT CTTTTTG-3' and antisense, 5'-AATTCAAAAAGAGATGCT GTCACTTAATAATCTCGAGATTATTAAGTGACAGCA TCTC-3'; KLK11-SH3 sense, 5'-CCGGCTGGTCTGTAA CCAGTCTCTTCTCGAGAAGAGACTGGTTACAGACCA GTTTTTG-3' and antisense, 5'-AATTCAAAAACTGGTCTG TAACCAGTCTCTTCTCGAGAAGAGACTGGTTACAGA CCAG-3'; control shRNA sense, 5'-CCGGAAACTACCGTT GTTATAGGTGTTCAAGAGACACCTATAACAACGGTA GTTTTTTTTG-3' and antisense, 5'-AATTCAAAAAAA ACTACCGTTGTTATAGGTGTCTCTTGAACACCTATA ACAACGGTAGTTT-3'. Lentiviral virions were produced by co-transfection of HEK293T cells with $5 \mu \mathrm{g}$ pLKO.1-puro vector and $5 \mu \mathrm{g}$ packaging and envelope vectors using Lipofectamine 2000 (Invitrogen) according to the manufacturer's protocol. Lentivirus was harvested $48 \mathrm{~h}$ after transfection. LOVO and HCT-8 cells were infected with lentivirus containing shKLK11 or SCR for $24 \mathrm{~h}$. Two days later, the virus-infected cells were selected by $2 \mu \mathrm{g} / \mathrm{ml}$ puromycin (Sigma-Aldrich; Merck KGaA, Darmstadt, Germany) for $48 \mathrm{~h}$ and subjected to required assays.
Cell viability assay. Cell viability was quantified using a 3-(4,5-dimethylthiazol-2-yl)-2,5-diphenyltetrazolium bromide (MTT) assay as previously described (22). Briefly, $3 \times 10^{3}$ transiently transfected LOVO and HCT- 8 cells (SCR or shKLK11) were seeded in 96-well plates and $20 \mu 1$ MTT solution $(5 \mathrm{mg} / \mathrm{ml}$; Sigma-Aldrich) was added to each well 72 and $96 \mathrm{~h}$ later. The optical density was measured using a microplate reader (Bio-Rad Laboratories, Inc., Hercules, CA, USA) at $595 \mathrm{~nm}$.

For drug sensitivity, cells were plated in 96-well plates at $5 \times 10^{4}$ cells per well, followed by treatment with 0,5 or $10 \mu \mathrm{mol} / \mathrm{lL}-\mathrm{OHP}$ for $24 \mathrm{~h}$. The optical density was then measured and the cell viability was calculated.

Annexin V-FITC apoptosis detection. Apoptosis detection was performed using an Annexin V Apoptosis Detection kit I (BD Biosciences, Franklin Lakes, NJ, USA). In brief, cells were collected and washed with phosphate-buffered saline (PBS). Then, $5 \mu \mathrm{l}$ annexin $\mathrm{V}$ and propidium iodide was added to the cell suspension and incubated at room temperature in the dark for $30 \mathrm{~min}$. The volume was then made up to $500 \mu \mathrm{l}$ and the cells were analyzed using a FACSCalibur flow cytometer (BD Biosciences).

Caspase-3 activity analysis. The activity of caspase-3 was measured using a Caspase-3 Assay kit (Abnova Corporation, Taipei, Taiwan) according to the manufacturer's instructions. In brief, $5 \times 10^{6}$ cells were harvested, resuspended in $50 \mu \mathrm{l}$ chilled cell lysis buffer and incubated on ice for $10 \mathrm{~min}$. Then, $50 \mu 1$ 2.0X Reaction Buffer was added to each sample, along with $5 \mu 1$ DEVD-pNA (4 mM) substrate and incubated for $2 \mathrm{~h}$ at $37^{\circ} \mathrm{C}$. The optical density was measured at $405 \mathrm{~nm}$ using a microplate reader (Bio-Rad Laboratories, Inc.).

Western blot analysis. Cell lysates were prepared in a buffer containing $50 \mathrm{mM}$ Tris- $\mathrm{HCl}(\mathrm{pH}$ 7.5), $1 \mathrm{mM}$ EDTA, $1 \% \mathrm{NP}-40(\mathrm{v} / \mathrm{v})$ and $150 \mathrm{mM} \mathrm{NaCl}$, supplemented with a mixture of complete protease inhibitors (Roche Diagnostics, Basel, Switzerland). Equal quantities of protein $(40 \mu \mathrm{g})$ were then separated on $10 \%$ SDS-PAGE and blotted onto a polyvinylidene difluoride membrane (Bio-Rad Laboratories, Inc.). Blocking was performed at room temperature using Tris-buffered saline with $0.1 \%$ Tween-20 (TBST; J\&K Chemical Ltd., Shanghai, China) containing 5\% non-fat milk for $1 \mathrm{~h}$. The membrane was then incubated with primary mouse monoclonal KLK11 antibody (sc-20387; 1:500) and rabbit polyclonal $\beta$-actin (sc-47778; 1:1,000; Santa Cruz Biotechnology, Inc., Dallas, TX, USA), Bcl-2 (\#2872; 1:1,000) and Bax (\#2772; 1:1,000) antibodies (Cell Signaling Technology, Inc., Danvers, MA, USA) in TBST at $4^{\circ} \mathrm{C}$ overnight and with the appropriate horseradish peroxidase-conjugated secondary antibody (CW0103M; 1:3000; $\mathrm{CWbiotech}$, Beijing, China) for $1 \mathrm{~h}$ at room temperature. Specific antibody binding was detected using an ECL system (GE Healthcare, Piscataway, NJ, USA).

Statistical analysis. Data are presented as the mean \pm standard deviation. Statistical analysis was performed using SPSS software, version 16.0 (SPSS, Inc., Chicago, IL, USA). Statistical significance was considered to be indicated by $\mathrm{P}<0.05$. 


\section{Results}

Stable knockdown of KLK11 specifically inhibits the expression of KLK11 mRNA and protein in CRC cells. A previous study has shown that the expression of KLK11 was upregulated in colorectal tumors (18). To determine whether KLK11 is involved in the progression of CRC, three different lentivirus-based shRNAs (KLK11 SH1, KLK11 SH2 and KLK11 SH3) were employed to downregulate KLK11 expression. The mRNA and protein levels of KLK11 in stably transfected LOVO and HCT-8 cells were confirmed using RT-qPCR and western blot analysis (Fig. 1). The mRNA expression levels of KLK11 were significantly decreased in KLK11 SH3 groups in both cell lines. The subsequent assays were performed with KLK11 SH3, which is furthermore referred to as shKLK11. These results suggested that the lentivirus-mediated shRNA targeting KLK11 could effectively knockdown KLK11 expression in CRC cells.

Downregulation of KLK11 inhibits growth and enhances apoptosis of $C R C$ cells. To determine the biological function of KLK11 in CRC progression, MTT assays were used to examine the proliferative ability of CRC cells. As shown in Fig. 2A and B, the proliferation rates of shKLK11-infected cells started to decrease and were reduced compared with those of the SCR groups on days 3 and 4.

There is considerable evidence indicating that apoptosis has a close association with cell growth (23). In the present study, apoptosis assays were performed using CRC cells following KLK11 silencing. The results of flow cytometric analysis indicated that the percentages of apoptotic cells were significantly increased in shKLK11-infected LOVO and HCT-8 cells compared with the respective SCR groups (Fig. 2C and D). This finding indicated that KLK11 may serve a crucial function in the proliferation and tumorigenesis of CRC cells in vitro.

Downregulation of KLK11 expression inactivates the apoptosis signaling pathway in CRC cells. To examine the mechanism underlying the inhibition of cell growth, the expression of Bcl-2 and Bax, two important proteins of the apoptosis signaling pathway (24) was investigated. Western blot analysis showed that knockdown of KLK11 lead to a reduction of $\mathrm{Bcl}-2$ and an increase of Bax in both cell lines (Fig. 3A). Caspase-3, a crucial mediator of apoptosis, is a frequently activated death protease, catalyzing the specific cleavage of various key cellular proteins (25). A significant increase in caspase-3 activity was detected in shKLK11-infected LOVO and HCT-8 cells compared with SCR groups (Fig. 3B). Collectively, these data demonstrated that the proliferative effect of KLK11 in CRC cells is regulated via the apoptosis pathway.

KLK11 silencing enhances sensitivity of CRC cells to L-OHP and L-OHP-induced apoptosis in vitro. Our previous study has shown that dysregulation of KLK11 expression had an association with FOLFOX4 chemotherapy in human CRC cells (26). However, whether KLK11 played a key role in affecting the sensitivity of CRC cells was not fully understood. To elaborate on this, LOVO and HCT-8 cells with stable
KLK11 silencing were treated with 0,5 or $10 \mu \mathrm{mol} / 1 \mathrm{~L}-\mathrm{OHP}$ for $24 \mathrm{~h}$. The results of MTT assay suggested that knockdown of KLK11 led to a significant reduction in the viability of $\mathrm{CRC}$ cells in response to L-OHP in a dose-dependent manner compared with control (Fig. 4A and B). Furthermore, flow cytometric analysis showed that the apoptotic rate of cells with stable KLK11 silencing treated with L-OHP was significantly higher than that of cells treated with control (Fig. 4C and D). Collectively, these results suggest that knockdown of KLK11 could increase the chemosensitivity of CRC cells to L-OHP by inducing apoptosis enhancement in vitro.

\section{Discussion}

Overexpression of KLK11 is a general feature in numerous human malignancies including $\mathrm{CRC}$, and the overexpression is often correlated with malignant behavior (27). In the present study, it was found that KLK11 silencing inhibited the growth and increased the apoptosis of CRC cells. Downregulation of KLK11 also increased caspase-3 activity by activating the apoptosis signaling pathway, which induced a reduction of the $\mathrm{Bcl}-2 /$ Bax ratio.

KLK11 is a member of the KLK family, which are dysregulated in multiple tumors (17). Previous experiments have shown that KLK11 was upregulated in malignant CRC tissues in comparison with noncancerous tissues, and was associated with highly invasive and positive nodal status (18). Therefore, we hypothesized that KLK11 might be an oncogene in colorectal tumors. As expected, lentivirus-mediated KLK11 silencing was able to effectively suppress the proliferation of colon cancer cells in vitro. Furthermore, knocking down KLK11 resulted in a significant upregulation of apoptosis in CRC cells. From these data, we suggest that KLK11 has a positive impact on the progression of CRC cells in vitro.

In order to determine the underlying mechanisms by which KLK11 is involved in cell growth, the present study analyzed apoptosis signaling in CRC cells. Bcl-2 and Bax, two crucial regulatory proteins that play important roles in the induction of apoptosis have been reported to regulate cancer growth $(28,29)$. The results of the present study indicated that KLK11 silencing may activate the apoptosis signaling pathway by increasing the expression level of Bax and decreasing the expression level of $\mathrm{Bcl}-2$, which induced a reduction of the $\mathrm{Bcl}-2 / \mathrm{Bax}$ ratio. Furthermore, the downregulation of KLK11 promotes caspase-3 activity, which results in the death of tumor cells.

There is considerable evidence supporting the hypothesis that mechanisms involved in resistance to chemotherapy correlate with apoptosis (30). The present study next investigated whether the KLK11 silencing was associated with sensitivity to L-OHP. Consistent with the above data, KLK11 silencing resulted in a higher inhibition of cell growth and apoptosis following exposure to L-OHP.

Based on these data, we conclude that knockdown of KLK11 could inhibit cell proliferation, induce apoptosis and increase the sensitivity of CRC cells to L-OHP in vitro, which may offer a novel therapeutic approach for L-OHP-resistant CRC treatment. Further studies are required to determine whether these findings are present in vivo. 
A

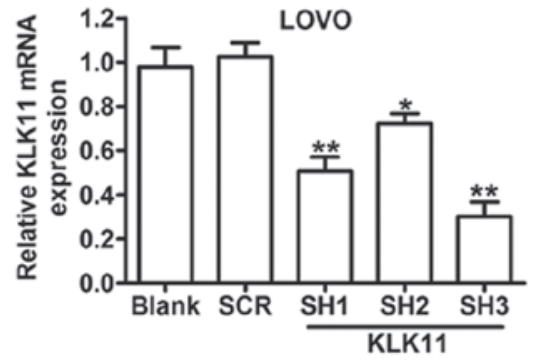

C

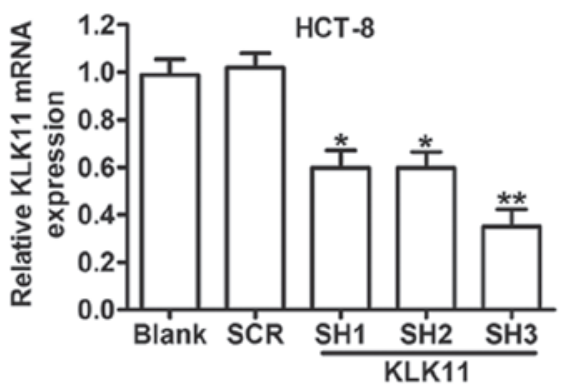

B

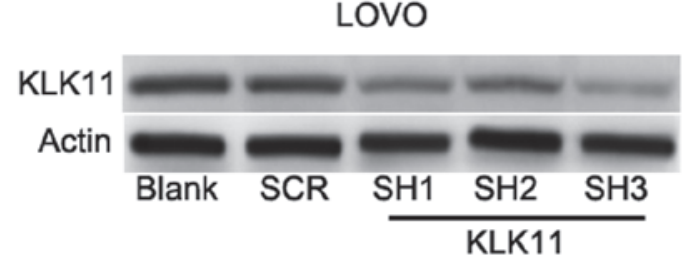

D

HCT-8

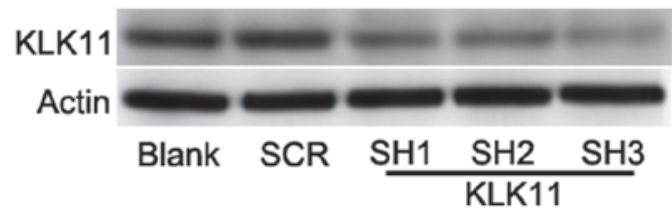

Figure 1. Establishment of cell lines that stably knockdown KLK11. Expression of (A) KLK11 mRNA and (B) KLK11 protein in LOVO cells and (C) KLK11 mRNA and (D) KLK11 protein in HCT-8 cells. mRNA levels were detected by reverse transcription-quantitative polymerase chain reaction, and protein levels by western blot analysis. $\beta$-actin was used as a loading control. ${ }^{*} \mathrm{P}<0.05,{ }^{* *} \mathrm{P}<0.01$ vs. SCR. KLK11, kallikrein 11; SCR, short hairpin RNA control.

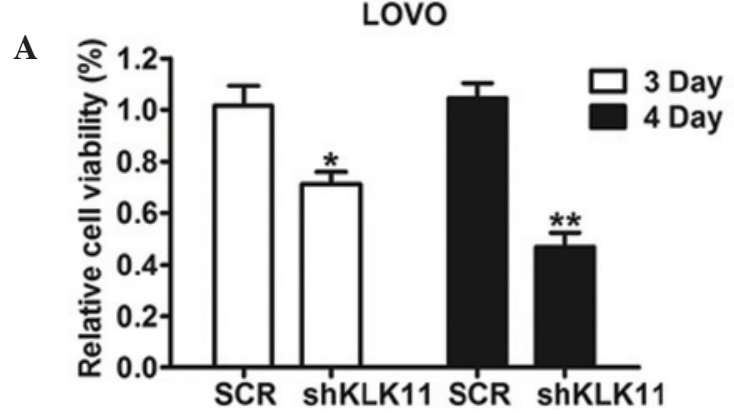

C
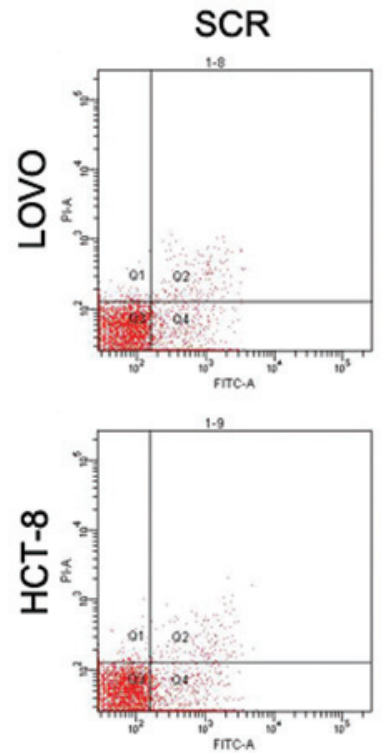

shKLK11
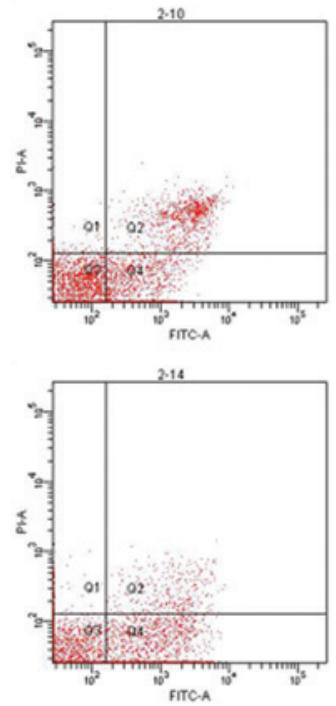

HCT-8

B

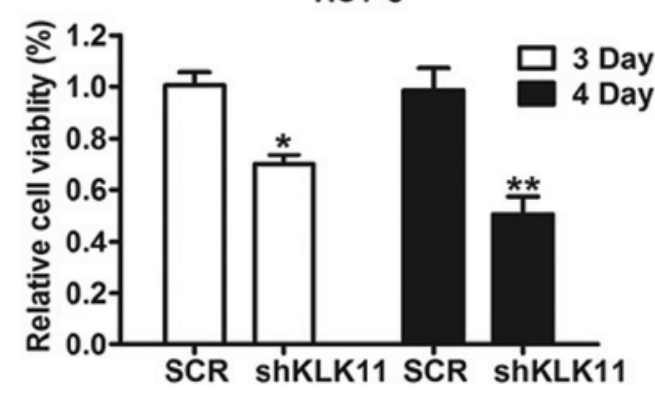

D

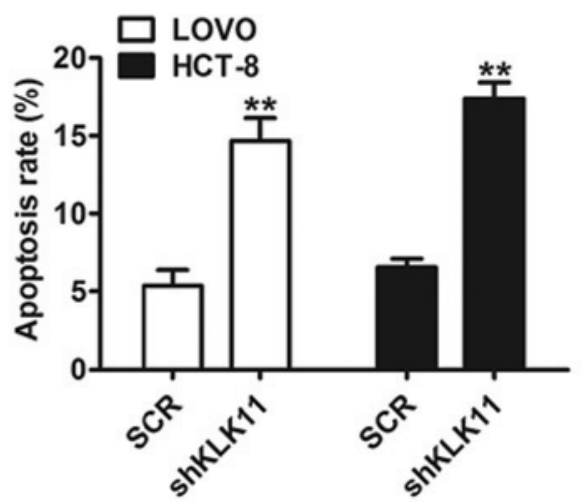

Figure 2. KLK11 shRNA inhibited cell proliferation and enhanced apoptosis. Proliferation levels of (A) LOVO and (B) HCT-8 cells after KLK11 silencing analyzed by 3-(4,5-dimethylthiazol-2-yl)-2,5-diphenyltetrazolium bromide assay. (C) Flow cytometric analysis of apoptosis in LOVO and HCT-8 cells transfected with SCR or shKLK11. (D) Quantification of the apoptotic cells. ${ }^{*} \mathrm{P}<0.05,{ }^{* *} \mathrm{P}<0.01$ vs. SCR. SCR, short hairpin RNA control; KLK11, kallikrein 11. 
A

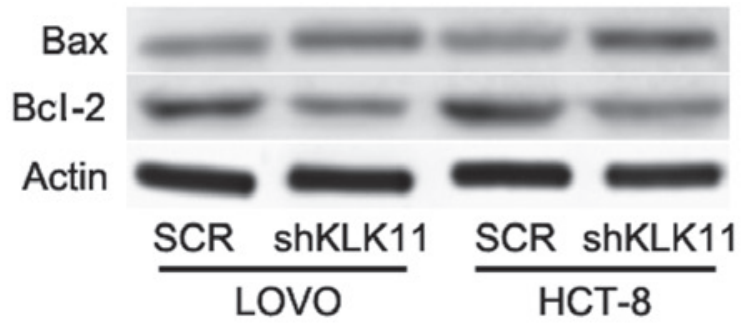

B

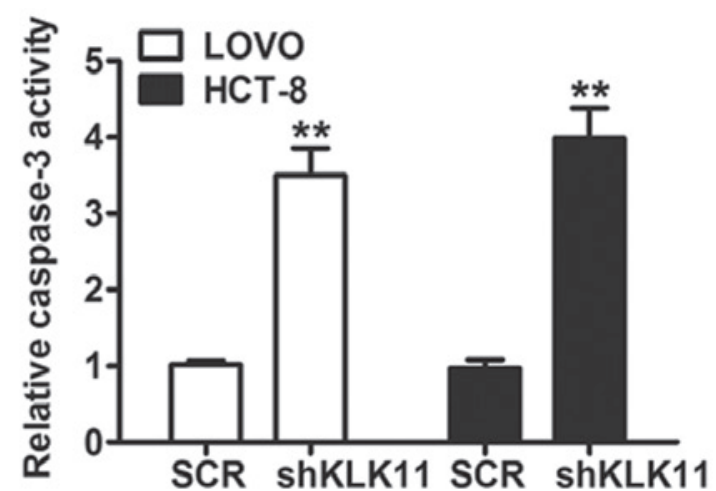

Figure 3. Effect of KLK11 silencing on the apoptosis signaling pathway. (A) Western blot analysis of Bcl-2 and Bax protein expression in LOVO and HCT-8 cells transfected with SCR or shKLK11. $\beta$-actin was used as a loading control. (B) Relative caspase-3 activity was determined in LOVO and HCT-8 cells transfected with SCR or shKLK11. ${ }^{* *} \mathrm{P}<0.01$ vs. SCR. Bcl-2, B-cell lymphoma 2; Bax, Bcl-2-associated protein X; SCR, short hairpin RNA control; KLK11, kallikrein 11.

A

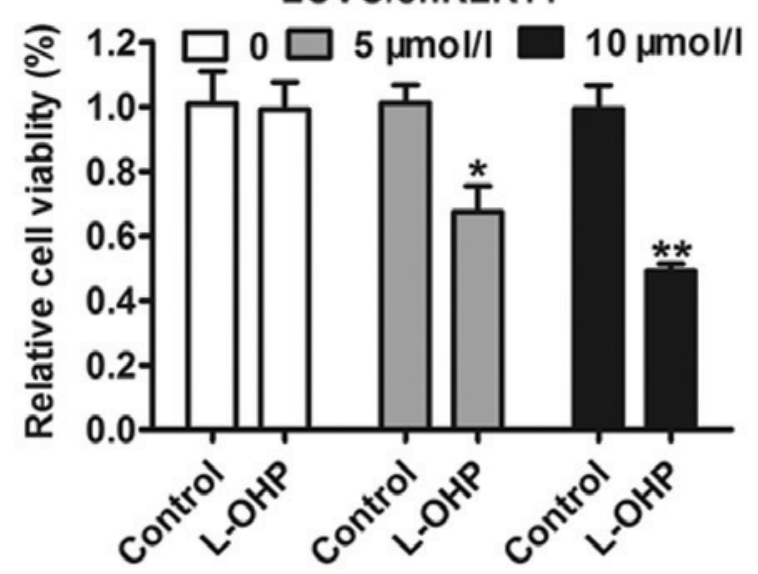

C
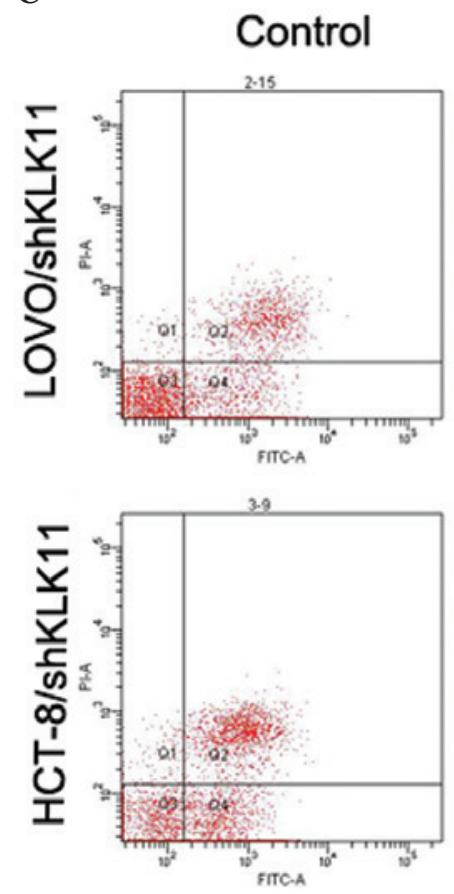

B

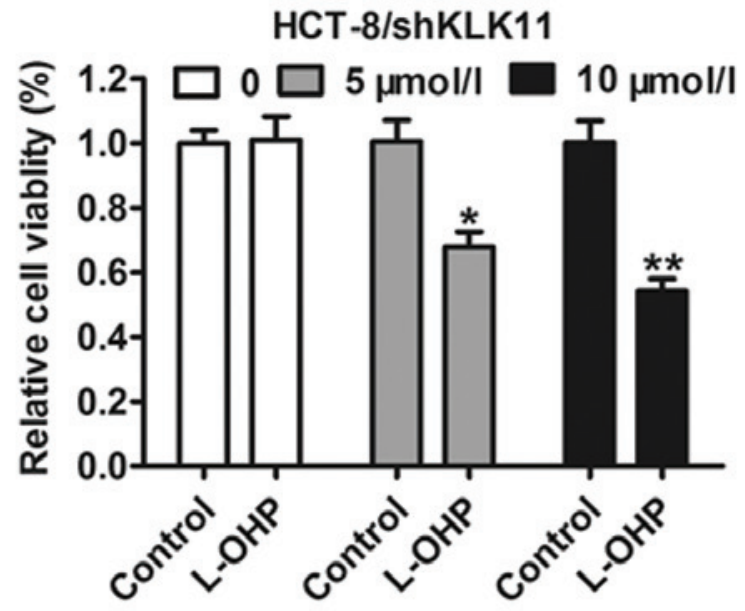

D

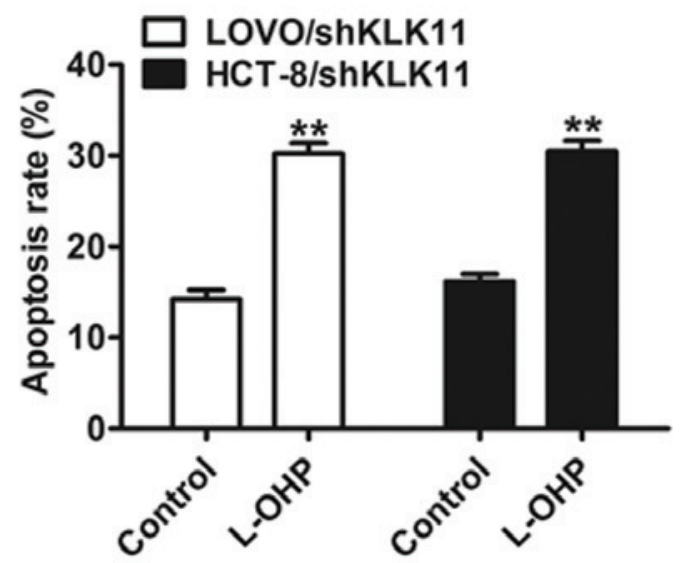

Figure 4. KLK11 silencing modulates the sensitivity to L-OHP. Viability of KLK11 knockdown (A) LOVO and (B) HCT-8 cells treated with the indicated concentrations of L-OHP for $24 \mathrm{~h}$. (C) The effect of L-OHP on apoptotic levels of the indicated cells analyzed by flow cytometry. (D) Quantification of the apoptotic cells. ${ }^{*} \mathrm{P}<0.05,{ }^{* *} \mathrm{P}<0.01$ vs. control. KLK11, kallikrein 11; L-OHP, oxaliplatin. 


\section{References}

1. Siegel R, Desantis C and Jemal A: Colorectal cancer statistics, 2014. CA Cancer J Clin 64: 104-117, 2014.

2. Talbot R and Kirkham S: Colorectal cancer. Lancet 376: 330; author reply 331-332, 2010.

3. Cunningham D, Atkin W, Lenz HJ, Lynch HT, Minsky B, Nordlinger B and Starling N: Colorectal cancer. Lancet 375: 1030-1047, 2010

4. Kim SY, Choi EJ, Yun JA, Jung ES, Oh ST, Kim JG, Kang WK and Lee SH: Syndecan-1 expression is associated with tumor size and EGFR expression in colorectal carcinoma: A clinicopathological study of 230 cases. Int J Med Sci 12: 92-99, 2015

5. Kuo IM, Huang SF, Chiang JM, Yeh CY, Chan KM, Chen JS and Yu MC: Clinical features and prognosis in hepatectomy for colorectal cancer with centrally located liver metastasis. World J Surg Oncol 13: 92, 2015.

6. Van Cutsem E, Nordlinger B, Adam R, Köhne CH, Pozzo C, Poston G, Ychou M and Rougier P; European Colorectal Metastases Treatment Group: Towards a pan-European consensus on the treatment of patients with colorectal liver metastases. Eur J Cancer 42: 2212-2221, 2006.

7. Díaz-Rubio E, Tabernero J, Gómez-España A, Massutí B, Sastre J, Chaves M, Abad A, Carrato A, Queralt B, Reina JJ, et al: Phase III study of capecitabine plus oxaliplatin compared with continuous-infusion fluorouracil plus oxaliplatin as first-line therapy in metastatic colorectal cancer: Final report of the Spanish Cooperative Group for the Treatment of Digestive Tumors Trial. J Clin Oncol 25: 4224-4230, 2007.

8. Cassidy J, Clarke S, Díaz-Rubio E, Scheithauer W, Figer A, Wong R, Koski S, Lichinitser M, Yang TS, Rivera F, et al Randomized phase III study of capecitabine plus oxaliplatin compared with fluorouracil/folinic acid plus oxaliplatin as first-line therapy for metastatic colorectal cancer. J Clin Oncol 26: 2006-2012,2008.

9. Nordlinger B, Sorbye H, Glimelius B, Poston GJ, Schlag PM, Rougier P, Bechstein WO, Primrose JN, Walpole ET, Finch-Jones M, et al: Perioperative chemotherapy with FOLFOX4 and surgery versus surgery alone for resectable liver metastases from colorectal cancer (EORTC Intergroup trial 40983): A randomised controlled trial. Lancet 371: 1007-1016, 2008.

10. Niitsu H, Hinoi T, Shimomura M, Egi H, Hattori M, Ishizaki Y, Adachi T, Saito Y, Miguchi M, Sawada H, et al: Up-front systemic chemotherapy is a feasible option compared to primary tumor resection followed by chemotherapy for colorectal cancer with unresectable synchronous metastases. World J Surg Oncol 13 : $162,2015$.

11. Tu J, Yu Y, Liu W and Chen S: Significance of human epidermal growth factor receptor 2 expression in colorectal cancer. Exp Ther Med 9: 17-24, 2015.

12. Tol J, Koopman M, Cats A, Rodenburg CJ, Creemers GJ, Schrama JG, Erdkamp FL, Vos AH, van Groeningen CJ, Sinnige HA, et al: Chemotherapy, bevacizumab and cetuximab in metastatic colorectal cancer. N Engl J Med 360: 563-572, 2009

13. Seki K, Tsuduki Y, Ioroi T, Yamane M, Yamauchi H, Shiraishi Y, Ogawa T, Nakata I, Nishiguchi K, Matsubayashi T, et al: Serum lactate dehydrogenase levels as a predictive marker of oxaliplatin-induced hypersensitivity reactions in Japanese patients with advanced colorectal cancer. Int J Med Sci 11: 641-645, 2014
14. Wang Q, Zhang C, Guo J, Huang J, Xi X, Zhang L and Cui X Super-compact treatment with a high dose of moxifloxacin in patients with drug-resistant tuberculosis and its resistance mechanisms. Exp Ther Med 9: 1314-1318, 2015.

15. Gan L, Lee I, Smith R, Argonza-Barrett R, Lei H, McCuaig J, Moss P, Paeper B and Wang K: Sequencing and expression analysis of the serine protease gene cluster located in chromosome 19q13 region. Gene 257: 119-130, 2000.

16. Nakamura T, Mitsui S, Okui A, Miki T and Yamaguchi N: Molecular cloning and expression of a variant form of hippostasin/KLK11 in prostate. Prostate 54: 299-305, 2003.

17. Mavridis K and Scorilas A: Prognostic value and biological role of the kallikrein-related peptidases in human malignancies. Future Oncol 6: 269-285, 2010.

18. Alexopoulou DK, Kontos CK, Christodoulou S, Papadopoulos IN and Scorilas A: KLK11 mRNA expression predicts poor disease-free and overall survival in colorectal adenocarcinoma patients. Biomark Med 8: 671-685, 2014.

19. Patsis C, Yiotakis I and Scorilas A: Diagnostic and prognostic significance of human kallikrein 11 (KLK11) mRNA expression levels in patients with laryngeal cancer. Clin Biochem 45: 623-630, 2012.

20. Unal D, Tasdemir A, Oguz A, Eroglu C, Cihan YB, Turak EE, Karaman $\mathrm{H}$ and Soyuer $\mathrm{S}$ : Is human kallikrein-11 in gastric cancer treated with surgery and adjuvant chemoradiotherapy associated with survival?. Pathol Res Pract 209: 779-783, 2013.

21. Livak KJ and Schmittgen TD: Analysis of relative gene expression data using real-time quantitative PCR and the 2(-Delta Delta C(T)) Method. Methods 25: 402-408, 2001

22. Zhu XC, Dong QZ, Zhang XF, Deng B, Jia HL, Ye QH, Qin LX and Wu XZ: microRNA-29a suppresses cell proliferation by targeting SPARC in hepatocellular carcinoma, Int J Mol Med 30: 1321-1326, 2012

23. Ouyang L, Shi Z, Zhao S, Wang FT, Zhou TT, Liu B and Bao JK: Programmed cell death pathways in cancer: A review of apoptosis, autophagy and programmed necrosis. Cell Prolif 45: 487-498, 2012.

24. Zhou F, Yang Y and Xing D: Bcl-2 and Bcl-xL play important roles in the crosstalk between autophagy and apoptosis. FEBS J 278: 403-413, 2011

25. Porter AG and Jänicke RU: Emerging roles of caspase-3 in apoptosis. Cell Death Differ 6: 99-104, 1999.

26. Li S, Lu X, Chi P and Pan J: Identification of HOXB8 and KLK11 expression levels as potential biomarkers to predict the effects of FOLFOX4 chemotherapy. Future Oncol 9: 727-736, 2013.

27. Wen YG, Wang Q, Zhou CZ, Yan DW, Qiu GQ, Yang C, Tang HM and Peng ZH. Identification and validation of Kallikrein-ralated peptidase 11 as a novel prognostic marker of gastric cancer based on immunohistochemistry. J Surg Oncol 104: 516-524, 2011.

28. Pettersson F, Dalgleish AG, Bissonnette RP and Colston KW: Retinoids cause apoptosis in pancreatic cancer cells via activation of RAR-gamma and altered expression of $\mathrm{Bcl}-2 / \mathrm{Bax} . \mathrm{Br}$ J Cancer 87: 555-561, 2002.

29. Yuan W, Xiaoyun H, Haifeng Q, Jing L, Weixu H, Ruofan D, Jinjin Y and Zongji S: MicroRNA-218 enhances the radiosensitivity of human cervical cancer via promoting radiation induced apoptosis. Int J Med Sci 11: 691-696, 2014.

30. Gillet JP and Gottesman MM: Mechanisms of multidrug resistance in cancer. Methods Mol Biol 596: 47-76, 2010. 